\title{
BMJ Open Migraine and traumatic brain injury: a cohort study in Taiwan
}

\author{
Qing-Rui Wang, ${ }^{1}$ Ying-Yi Lu, ${ }^{2,3,4}$ Ying-Ju Su, ${ }^{5}$ Hao Qin, ${ }^{6}$ Li Zhang, ${ }^{7}$ Ming-Kung Wu, ${ }^{8}$ \\ Cong-Liang Zhang, ${ }^{9}$ Chieh-Hsin $\mathrm{Wu}^{10,11}$
}

To cite: Wang Q-R, Lu Y-Y, Su Y-J, et al. Migraine and traumatic brain injury: a cohort study in Taiwan. BMJ Open 2019;9:e027251. doi:10.1136/ bmjopen-2018-027251

- Prepublication history for this paper is available online. To view these files please visit the journal online (http://dx.doi org/10.1136/bmjopen-2018027251).

Received 13 October 2018

Revised 25 June 2019

Accepted 04 July 2019
Check for updates

(C) Author(s) (or their employer(s)) 2019. Re-use permitted under CC BY-NC. No commercial re-use. See rights and permissions. Published by BMJ.

For numbered affiliations see end of article.

Correspondence to Professor Chieh-Hsin Wu; wujoeys@gmail.com

\begin{abstract}
Objective Traumatic brain injury is now a major contributor to the global healthcare burden. Migraine is another debilitating disease with a global health impact. While most researchers agree that traumatic brain injury is a risk factor for migraine, whether migraine is a risk factor for traumatic brain injury still remains under debate. We therefore aimed to investigate whether migraine was a risk factor for developing traumatic brain injury.

Study design Retrospective population-based cohort study.

Setting Data for people who had been diagnosed with migraine were retrieved from Taiwan's National Health Insurance Research Database.

Participants We identified 7267 patients with newly diagnosed migraine during 1996-2010. The migraineurs to non-migraineurs ratio was set at 1:4 to enhance the power of statistical tests.

Primary and secondary outcome measures We used multivariate Cox proportional hazard regression models to assess the effects of migraines on the risk of traumatic brain injury after adjusting for potential confounders. Results The overall traumatic brain injury risk was 1.78 times greater in the migraine group compared with the non-migraine group after controlling for covariates. Additionally, patients with previous diagnoses of alcoholattributed disease, mental disorders and diabetes mellitus had a significantly higher traumatic brain injury risk compared with those with no history of these diagnoses. Conclusions This study of a population-based database indicated that migraine is a traumatic brain injury risk factor. Greater attention to migraine-targeted treatment modalities may reduce traumatic brain injury-related morbidity and mortality.
\end{abstract}

\section{INTRODUCTION}

Globally, an estimated 10 million people suffer a traumatic brain injury (TBI) event every year. ${ }^{1}$ The combined incidence of TBI-related emergency department visits, inpatient hospital admissions and mortalities reached 823.7 per 100000 in $2010 . .^{2}$ Additionally, TBI is a major cause of long-term disability in young adults ${ }^{134}$ and can negatively affect quality of life in patients, their friends and relatives, and even the general population..$^{5-7}$ By 2020, TBI is projected to be the third largest contributor to the global healthcare burden. ${ }^{8-10}$ Therefore, an
Strengths and limitations of this study

- This is the first observational cohort study using a Chinese population-based data set in Taiwan to clarify whether migraine is a risk factor for traumatic brain injury.

- The major strength of this research is the use of a large population-based database, which could provide sufficient statistical power.

- The secondary database used in the study was encrypted to protect citizen's privacy.

- Self- reported pain scores for rating migraine were not recorded in the database.

- The results of the research need to be interpreted with caution in different ethnicities.

improved understanding of pathophysiology of TBI and its risk factors is needed to minimise the risk of TBI-related morbidities and mortalities in high-risk groups for TBI.

Migraine is a debilitating disease that has a significant impact on worldwide health. ${ }^{11}$ According to the primary criteria for headache, a migraine headache is defined as a moderate to severe unilateral headache that may be accompanied by photophobia, vomiting or nausea, and visual symptoms. Migraines can also compromise cognition, vestibular function, emotions and social behaviours. ${ }^{1213}$ Thus, the debilitating effects of migraine can make patients prone to TBI. Most studies of associations between TBI and migraine have focused on causal relationships between TBI and post-traumatic migraine. While most researchers agree that TBI is a risk factor for migraine,${ }^{12}$ whether migraine is a risk factor for TBI remains unclear. For example, although Gordon et al reported that migraine is a risk factor for TBI, the coding procedures used in their study were not available for further validation. Another problem is that the study results vary because data reviewed by Gordon et al were collected from questionnaires. Additionally, the psychopathology of TBI has not been precisely ascertained. ${ }^{14}$ Therefore, Gordon et al provide little information about the 
association between migraine and TBI. Current policies for TBI care and prevention can be highly burdensome for patients, their relatives and society. Given the limited availability of epidemiological data for TBI, the objective of this study was to conduct a nationwide study of patients with migraine to clarify the association between TBI and migraine.

\section{METHODS}

\section{Data sources}

\section{Methods}

\section{Database}

This population-based cohort study was performed using computerised records from the Taiwan National Health Insurance Research Database (NHIRD), a large encrypted secondary organised collection of data maintained by the Taiwan national healthcare system. More than $99 \%$ of the population (23.74 million) in Taiwan have been covered by the single-payer National Health Insurance since its implementation in 1995. The government entrusts the Taiwan National Health Research Institute with maintaining this database by continuously collecting relevant registration and claims data for the purpose of academic research. Therefore, the NHIRD contains comprehensive medical records of literally the entire population of Taiwan for the past two decades. This retrospective cohort study analysed a subset of the NHIRD, the Longitudinal Health Insurance Database 2010, which contains detailed data for 1 million individuals sampled from the primary NHIRD. International Classification of Diseases, Ninth Revision, Clinical Modification (ICD-9-CM) codes were used to identify and classify diseases. ${ }^{15} 16$ The study was performed in accordance with the Declaration of Helsinki guidelines.

\section{Study population}

The migraine cohort in this study included 7267 patients diagnosed with migraine (ICD-9-CM code 346) in 1996-2010 and aged 18 years or older. To maximise case ascertainment, the analysis was limited to patients with a record of $\geq 1$ migraine diagnosis during inpatient service or $\geq 2$ migraine diagnoses during outpatient visits; additionally, only records in which an ICD-9-CM code for migraine had been assigned by a neurologist were used. The date of the first migraine diagnosis was defined as the index date. Migraine with aura, migraine without aura and unspecified migraine were defined according to ICD-9-CM codes 346.0, 346.1 and 346.9, respectively. ${ }^{17} \mathrm{~A}$ TBI (ICD-9-CM code 800803-804 and 850-854; operation codes $01.23,01.24,01.25,0131,01.39$ and 02.01$)^{1518-21}$ was defined as a record of two or more TBI diagnoses in outpatient visits or one or more TBI diagnoses in inpatient admissions. Cases of TBI were further categorised into three groups based on the severity of injury. A TBI was classified as severe if the patient required surgery in the course of inpatient treatment; a TBI was classified as moderate if the patient required hospitalisation but not surgery; a TBI was classified as mild if the patient did not require inpatient treatment. ${ }^{152}$

The exclusion criteria were diagnosis of TBI before or on the index date, age younger than 18 years and incomplete information. The date of migraine diagnosis was defined as the date of the first diagnosis of migraine. An identical 'pseudo date of diagnosis' was assigned to matching control cases, which was the date of diagnosis in matched subjects.

The migraine to non-migraine ratio was set at $1: 4$ to obtain a sufficient number of TBI cases and to enhance the power of statistical tests used in stratified analyses. Each patient in the migraine cohort was matched by gender, age and year of migraine diagnosis with four persons in the non-migraine cohort obtained in the same period. Therefore, 29068 cases were enrolled in the non-migraine cohort. Figure 1 shows a flow chart of the study procedure.

\section{Outcome and comorbidities}

Both the migraine and the control cohorts were followed up until the end of 2010, withdrawal from insurance or TBI diagnosis, whichever occurred first. Baseline comorbidities which were ascertained from ICD-9-CM codes in claims records included diabetes mellitus (ICD-9-CM code 250), hyperlipidaemia (ICD-9-CM code 272), hypertension (ICD-9-CM codes 401-405), coronary artery disease (ICD-9-CM codes 410-414), liver cirrhosis (ICD-9-CM code 571), tobacco use disorder (ICD-9-CM code 350.1), obesity (ICD-9-CM code 278), mental disorders (ICD-9-CM codes 290-319) and alcohol-attributed diseases (ICD-9-CM codes 291.0-9, 303, 305.0, 357.5, 425.5, 535.3, 571.0-3980.0 and V11.3). At follow-up, income-related insurance payments and urbanisation indices were used as proxy measures of individual socioeconomic status. Insurance premiums were classified into three groups as stated by monthly insurance payments: less than NT\$20 000; NT\$20000-NT\$40 000; and more than NT $\$ 40000 .{ }^{23}{ }^{24}$ Urbanisation index was categorised as high (metropolitan cities), medium (small cities and suburban areas) and low (rural areas). ${ }^{25}$

\section{Statistical analyses}

The clinical characteristics and categorical demographics of the study cases were analysed by the $\chi^{2}$ test to compare between the migraine and non-migraine cohorts. KaplanMeier method was used to estimate cumulative incidence of TBI, and the differences between the curves were calculated by two-tailed log-rank tests. For patients with migraine, survival was calculated until the study endpoint (31 December 2010), until the date of inpatient hospital treatment or until the date of an outpatient visit for TBI, whichever occurred first. The two cohorts were compared using the TBI incidence rates estimated in 1000 person-years. Mean age and follow-up time (years) were compared between the two cohorts by using Student's t-test and Wilcoxon rank-sum test. If the proportional hazards assumption was satisfied, univariable 


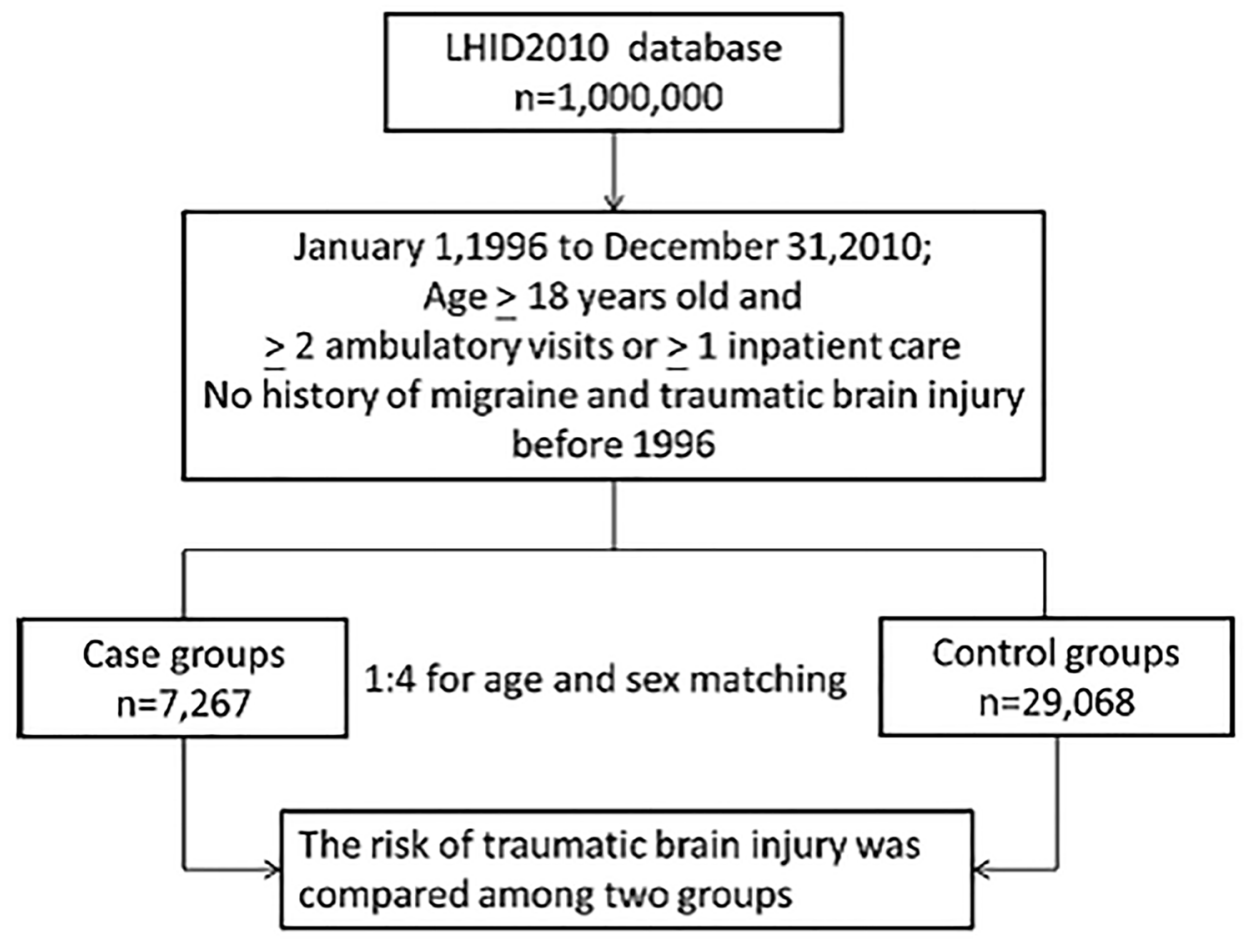

Figure 1 Flow diagram summarising the process of enrolment. LHID, Longitudinal Health Insurance Database.

and multivariable Cox proportional hazard regression models were performed to calculate HRs and $95 \%$ CIs for TBI. $^{26-29}$ All statistical tests were performed using Statistical Analysis Software V.9.4 (SAS Institute), and adopted a two-tailed significance level of 0.05 .

\section{Patient and public involvement}

The patients and the public were not involved in the research.

\section{RESULTS}

Baseline characteristics of patients with and without migraine In table 1, the baseline demographic characteristics and comorbidities of the study participants are shown. In the migraine group, $73.84 \%$ patients were female. Compared with the non-migraine group, the migraine cohort had significantly higher percentages of patients with diabetes mellitus $(31.64 \%$ vs $16.61 \%$; $\mathrm{p}<0.001)$, hypertension (50.14\% vs $25.04 \%$; $<<0.001)$, hyperlipidaemia $(50.28 \%$ vs $25.76 \% ; \mathrm{p}<0.001)$, coronary artery disease $(8.52 \%$ vs $2.49 \%$; $<<0.001)$, liver cirrhosis $(48.20 \%$ vs $25.14 \%$; $\mathrm{p}<0.001)$, mental disorders $(31.48 \%$ vs $11.34 \%$; $<<0.001)$, obesity $(4.66 \%$ vs $2.22 \%$; $<<0.001)$, alcohol-attributed disease $(4.14 \%$ vs $2.13 \% ; \mathrm{p}<0.001)$ and tobacco use disorder $(4.32$ vs $0.65 ; \mathrm{p}<0.001)$. During a median observation time of 2.8 years, $3.74 \%$ (272) of the patients with migraine had TBI (IQR=1.2-5.4). The TBI incidence in the migraine cohort was significantly $(\mathrm{p}<0.001)$ higher than that in the non-migraine cohort ( 797 with TBI out of
29068 age and gender-matched controls (2.74\%)) during a median observation time of 5.3 years $(\mathrm{IQR}=2.6-8.4)$. The median duration of follow-up of TBI was significantly shorter in the migraine group (2.8 years) compared with the non-migraine group ( 5.3 years).

\section{TBI incidence and risk}

Table 2 shows the results of the stratification analysis, including TBI incidence and HRs by age, gender and comorbidity. During the follow-up period, TBI developed in $3.74 \%$ (272) of the patients with migraine and in $2.74 \%$ (797) of the non-migraine patients. The overall TBI risk was 1.78 times greater in the migraine group compared with the non-migraine group (5.35 vs 1.86 per 1000 person-years, respectively) after adjusting for age, gender, income, urbanisation and related comorbidities (hypertension, diabetes mellitus, hyperlipidaemia, coronary artery disease, liver cirrhosis, mental disorders, obesity, alcohol-attributed disease and tobacco use disorder).

In both cohorts, gender-specific analyses showed that the incidence of migraine per 1000 person-years was higher in men than in women (6.46 vs 4.97 , respectively, in the migraine cohort; 2.59 vs 1.60 , respectively, in the non-migraine cohort). The migraine group had a significantly higher TBI risk in both genders (adjusted $\mathrm{HR}=1.94$, 95\% CI 1.60 to 2.36 for women; adjusted $\mathrm{HR}=1.48,95 \%$ CI 1.13 to 1.95 for men). In all age groups, the migraine cohort consistently showed a higher incidence of TBI, and the incidence rate increased with age. Age-specific 
Table 1 Baseline characteristics of patients with and without migraine

\begin{tabular}{|c|c|c|c|}
\hline \multirow[b]{2}{*}{ Variables } & \multicolumn{2}{|l|}{ Migraine } & \multirow[t]{2}{*}{$\mathbf{P}$ value } \\
\hline & Yes $(n=7267)$ & No $(n=29068)$ & \\
\hline Newly diagnosed traumatic brain injury, $\mathrm{n}(\%)$ & $272(3.74)$ & $797(2.74)$ & $<0.001$ \\
\hline $\begin{array}{l}\text { Period of developing traumatic brain injury, years (median, } \\
\text { IQR) }\end{array}$ & $2.8(1.2-5.4)$ & $5.3(2.6-8.4)$ & $<0.001$ \\
\hline Mean age at enrolment (years, SD) & $47.2(14.6)$ & $47.1(14.8)$ & 0.565 \\
\hline \multicolumn{4}{|l|}{ Age group, n (\%) } \\
\hline $18-49$ & $4341(59.74)$ & $17364(59.74)$ & \\
\hline$\geq 50$ & $2926(40.26)$ & $11704(40.26)$ & 1.000 \\
\hline \multicolumn{4}{|l|}{ Gender, n (\%) } \\
\hline Men & $1901(26.16)$ & $7604(26.16)$ & \\
\hline Women & $5366(73.84)$ & $21464(73.84)$ & 1.000 \\
\hline \multicolumn{4}{|l|}{ Income, n (\%) } \\
\hline Low $(<\mathrm{NT} \$ 20000)$ & $5493(75.59)$ & 22504 (77.42) & \\
\hline Medium (NT\$20 000-NT\$39 999) & $1216(16.73)$ & $4542(15.63)$ & \\
\hline High (>NT\$40 000) & $558(7.68)$ & $2022(6.96)$ & $<0.001$ \\
\hline \multicolumn{4}{|l|}{ Degree of urbanisation, $\mathrm{n}(\%)$} \\
\hline Urban & $4385(60.34)$ & $16745(57.61)$ & \\
\hline Suburban & $2350(32.34)$ & $10018(34.46)$ & \\
\hline Rural & $532(7.32)$ & $2305(7.93)$ & $<0.001$ \\
\hline \multicolumn{4}{|l|}{ Comorbidity, n (\%) } \\
\hline Hypertension & $3644(50.14)$ & $7280(25.04)$ & $<0.001$ \\
\hline Diabetes mellitus & $2299(31.64)$ & $4829(16.61)$ & $<0.001$ \\
\hline Hyperlipidaemia & $3654(50.28)$ & $7488(25.76)$ & $<0.001$ \\
\hline Coronary artery disease & $619(8.52)$ & $723(2.49)$ & $<0.001$ \\
\hline Liver cirrhosis & $3503(48.20)$ & $7307(25.14)$ & $<0.001$ \\
\hline Mental disorders & $2288(31.48)$ & 3297 (11.34) & $<0.001$ \\
\hline Obesity & $339(4.66)$ & $645(2.22)$ & $<0.001$ \\
\hline Tobacco use disorder & 314 (4.32) & $188(0.65)$ & $<0.001$ \\
\hline Alcohol-attributed disease & $301(4.14)$ & $619(2.13)$ & $<0.001$ \\
\hline
\end{tabular}

risk comparisons also showed that, for all age groups, the migraine cohort had a significantly higher TBI risk compared with the non-migraine cohort. Without considering comorbidities, TBI risk was lower in non-migraine patients than in patients with migraine. However, the TBI risk in patients with migraine with comorbidity was lower than the TBI risk in non-migraine patients with comorbidity.

In figure 2, the cumulative incidence of TBI between the migraine and non-migraine groups is compared using the Kaplan-Meier curves at the 15-year follow-up. The KaplanMeier curves revealed a significantly higher cumulative incidence of TBI in the migraine cohort compared with the non-migraine cohort (log-rank test, $\mathrm{p}<0.001)$.

Risk factors for TBI in patients with migraine

Table 3 shows the Cox regression analysis results, which revealed that risk factors for TBI in the migraine group were alcohol-attributed disease (adjusted $\mathrm{HR}=2.45 ; 95 \%$ CI
1.67 to 3.60 ), mental disorders (adjusted $\mathrm{HR}=1.42 ; 95 \% \mathrm{CI}$ 1.11 to 1.81 ) and diabetes mellitus (adjusted HR=1.46; $95 \%$ CI 1.15 to 1.85 ). Notably, female gender and high income had protective roles against TBI in these patients.

Table 4 compares TBI risk in different migraine types. For all migraine types, the migraine group had a significantly higher TBI risk compared with the non-migraine group.

Table 5 compares incidence rates and HRs for various outcomes between patients with and without migraine. Compared with the non-migraine cohort, the migraine cohort faced 149\% increase in the risk of mild TBI, 36\% increase in the risk of moderate TBI and 263\% increase in the risk of severe TBI after adjustment of covariates. Migraine had the strongest association with severe TBI.

\section{DISCUSSION}

To the best of our knowledge, this study is the first to investigate TBI risk in a large cohort of patients with 
Table 2 Incidence and HRs of traumatic brain injury by demographic characteristics, comorbidity and follow-up duration among patients with or without migraine

\begin{tabular}{|c|c|c|c|c|c|c|}
\hline \multirow[b]{2}{*}{ Variables } & \multicolumn{2}{|c|}{ Patients with migraine } & \multicolumn{2}{|c|}{ Patients without migraine } & \multicolumn{2}{|c|}{ Compared with non-migraine } \\
\hline & TBI & Rate & TBI & Rate & Crude HR* $(95 \% \mathrm{Cl})$ & Adjusted HR* $(95 \% \mathrm{Cl})$ \\
\hline Overall & 272 & 5.35 & 797 & 1.86 & $2.26(1.96$ to 2.61$) \dagger$ & $1.78(1.52$ to 2.08$) \dagger$ \\
\hline Men & 84 & 6.46 & 289 & 2.59 & $1.96(1.53$ to 2.51$) \dagger$ & $1.48(1.13$ to 1.95$) \ddagger$ \\
\hline Women & 188 & 4.97 & 508 & 1.60 & 2.44 (2.05 to 2.89$) \dagger$ & 1.94 (1.60 to 2.36$) \dagger$ \\
\hline \multicolumn{7}{|c|}{ Stratified by age } \\
\hline $18-49$ & 149 & 4.77 & 422 & 1.66 & 2.29 (1.89 to 2.77$) \dagger$ & 1.85 (1.51 to 2.26$) \dagger$ \\
\hline$\geq 50$ & 123 & 6.29 & 375 & 2.18 & $2.25(1.83$ to 2.77$) \dagger$ & $1.71(1.37$ to 2.13$) \dagger$ \\
\hline \multicolumn{7}{|c|}{ Comorbidity§ } \\
\hline No & 28 & 4.39 & 286 & 1.38 & 2.42 (1.64 to 3.58$) \dagger$ & 2.49 (1.69 to 3.69$) \dagger$ \\
\hline
\end{tabular}

Rate refers to incidence rate per 1000 person-years.

*Model adjusted for age, gender, income, urbanisation level and relevant comorbidities (hypertension, diabetes mellitus, hyperlipidaemia, coronary artery disease, liver cirrhosis, mental disorders, obesity, tobacco use disorder and alcohol-attributed disease).

$\dagger P<0.001$.

$\ddagger \mathrm{P}=0.005$.

§Patients with any examined comorbidities, including hypertension, diabetes mellitus, hyperlipidaemia, coronary artery disease, liver cirrhosis, mental disorders, obesity, tobacco use disorder and alcohol-attributed disease, were classified as the comorbidity group.

$\mathrm{TBI}$, traumatic brain injury.

migraine in Asia. The main finding of this study is that migraine is a potential risk factor for subsequent TBI. Specifically, the migraine group had a 1.78-fold higher TBI risk compared with the non-migraine control group after adjusting for age, income, gender, urbanisation and related medical comorbidities. The association between migraine and TBI risk was significantly higher in younger populations compared with older populations analysed in this study. Additionally, TBI risk was significantly higher in patients who had a previous diagnosis of alcohol-attributed disease, mental disorders or diabetes mellitus compared with those with no history of these diagnoses. Notably, patients in the migraine group were prone to severe TBI.

Previous studies have similarly shown that migraine is associated with TBI. For example, ${ }^{30}$ a multivariate analysis in an exploratory study by Gordon $e t$ al reported that independent risk factors for concussion include migraine, young age and male gender. Notably, their study analysed the publicly available set of microdata collected by the Canadian Community Health Survey of 270 patients (age, 12-24 years) who had a history of sports-related concussion. The data set was remarkably complete, for example, it had less than $0.5 \%$ missing data and no missing age or gender data for migraine and TBI. However, the study only analysed self-reported data for migraine history and did not provide information regarding the cause-effect relationship between migraine and TBI. The patients in the Canadian Community Health Survey were also considerably younger than those in our current study and were not matched by gender. Furthermore, the sample was not sufficiently representative of the general population or of true clinical status. ${ }^{14}$ In 2009, Lau et al reported a study of the prognostic value of self-reported symptoms and neurocognitive performance after concussion in a group of 108 football players aged 13-19 years. Fifteen participants reported a preinjury migraine headache history, and 23 participants said they had a history

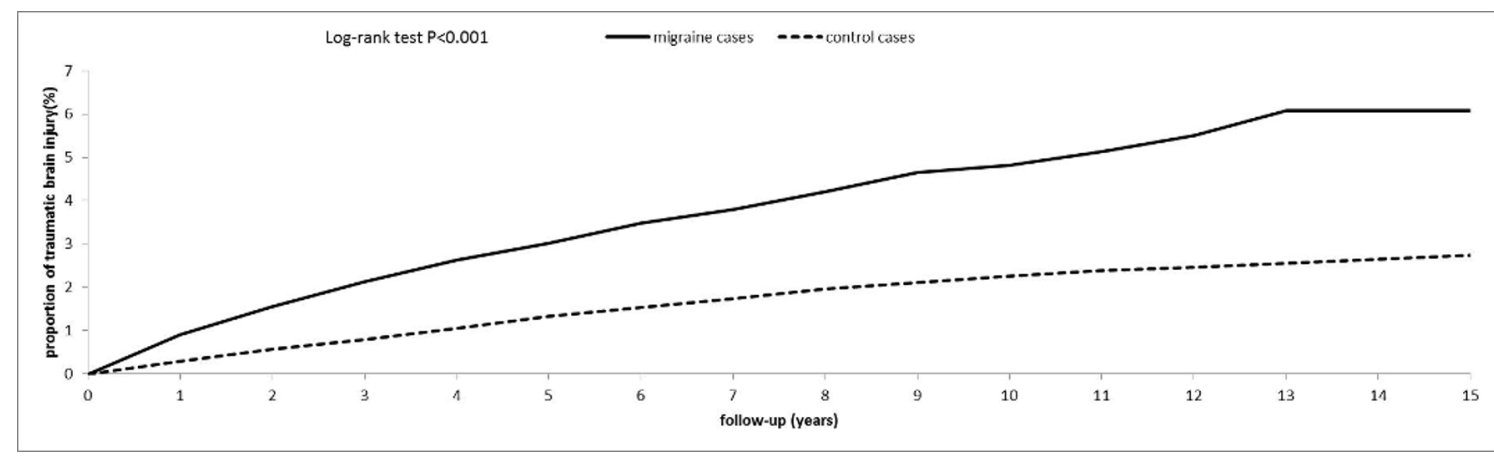

Figure 2 Cumulative incidence of traumatic brain injury among patients with migraine and the control cohort. 
Table 3 Cox regression model: significant predictors of TB after migraine

\begin{tabular}{lllc}
\hline Variables & Adjusted HR* & $(95 \% \mathbf{C l})$ & P value \\
\hline $\begin{array}{l}\text { Alcohol- } \\
\text { attributed } \\
\text { disease }\end{array}$ & 2.45 & $(1.67$ to 3.60$)$ & $<0.001$ \\
$\begin{array}{l}\text { Mental disorders } \\
\text { Diabetes }\end{array}$ & 1.42 & $(1.11$ to 1.81$)$ & 0.005 \\
$\begin{array}{l}\text { mellitus } \\
\text { Female gender }\end{array}$ & 0.72 & $(1.15$ to 1.85$)$ & 0.002 \\
\hline High income & 0.52 & $(0.56$ to 0.94$)$ & 0.016 \\
\hline
\end{tabular}

The adjusted $\mathrm{HR}$ and $95 \% \mathrm{Cl}$ were estimated by a stepwise Cox proportional hazards regression method

*Model adjusted for age, gender, income, urbanisation level and relevant comorbidities (hypertension, diabetes mellitus, hyperlipidaemia, coronary artery disease, liver cirrhosis, mental disorders, obesity, tobacco use disorder and alcohol-attributed disease).

$\mathrm{TBI}$, traumatic brain injury.

of either migraine or other headache types. However, no causal relationship was established. ${ }^{31}$

Moreover, reported causes of migraine include failure of cerebral ion homeostatic regulation and excess release of excitatory amino acids caused by cortical spreading suppression and depolarisation. ${ }^{32}$ Breakdown of ion homeostasis caused by cortical spreading suppression is the primary mechanism of neuronal damage after TBI. Although migraine and TBI share some common pathophysiologies, ${ }^{32} 33$ the exact mechanism of the association between preinjury migraine and TBI is not well understood.

Although the exact mechanisms underlying the relationship between migraine and TBI are unclear, several risk factors for TBI in patients with migraine are established. First, migraine is associated with lower cognitive function. For example, a 2014 study by Gil-Gouveia et al systematically reviewed evidence of decline in cognitive function during a migraine attack. Their review showed that migraine usually causes deficits in executive functions such as attention, working memory and processing speed. ${ }^{34}$ Another nationwide study by Chuang et al reported that migraine is associated with an increased risk of future dementia after adjusting for comorbidities. The migraine and non-migraine cohorts in their study included 33468 and 133872 patients, respectively, and had similar distributions of age and gender. The authors concluded that the migraine cohort had a 1.33fold higher dementia risk compared with controls. ${ }^{35}$ Finally, migraine is associated with low cognitive function and impaired executive control functions, ${ }^{36}$ which can increase the risk of accidents or TBI by increasing risky behaviour or carelessness. ${ }^{37}$

A second cause of increased TBI risk in patients with migraine is their increased likelihood of psychiatric comorbidities, ${ }^{38-40}$ which have been identified as a TBI risk factor. ${ }^{41}{ }^{42}$ For example, a review of a general population of patients by Antonaci et al found that patients with migraine have a higher risk of affective and anxiety disorders compared with those without migraine. Another line of research suggests that migraine is associated with bipolar disorder. One hypothesis is that people with mental disorders have lower than normal attention, concentration and reaction times, all of which contribute to increased TBI risk. ${ }^{38434}$ Third, chronic migraine headache causes postural instability and vestibulopathy, which increase the risk of falls; falls are the major cause of TBI in the USA. ${ }^{45}$

The main strength of this study is the use of a population-based data set, which included nearly all cases of migraine and TBI treated in Taiwan during the study period, to demonstrate an association between migraine and TBI risk. The cohort study design with controls and the large sample size also enabled stratification of analyses by the severity of TBI and time. Since data collection was not affected by recall bias, the analyses had sufficient statistical power to identify significant differences between the migraine cohort and non-migraine cohorts.

However, several weaknesses of this study are noted. The first limitation is potential coding errors. Like most administrative databases, coding information is directly generated from administrative discharge claims data designed for billing purposes. Clinical physicians are subject to oversights in documentation and entering diagnostic codes. However, the validity of true diagnoses was

Table 4 Incidence and HRs for TBI stratified by the different types of migraine

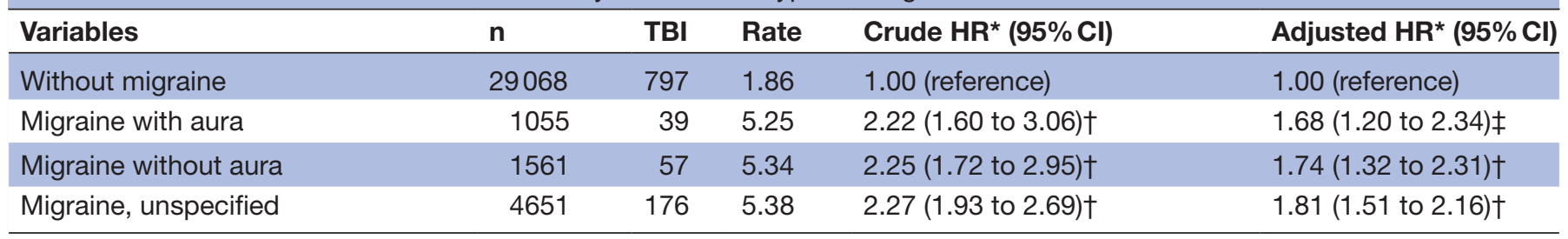

Rate refers to incidence rate per 1000 person-years.

*Model adjusted for age, gender, income, urbanisation level and relevant comorbidities (hypertension, diabetes mellitus, hyperlipidaemia, coronary artery disease, liver cirrhosis, mental disorders, obesity, tobacco use disorder and alcohol-attributed disease).

$\dagger P<0.001$

$\ddagger P=0.002$.

$\mathrm{TBI}$, traumatic brain injury. 
Table 5 Incidence and HRs of different severity of TBI risk in patients with migraine compared with those without migraine

\begin{tabular}{|c|c|c|c|c|c|c|}
\hline \multirow{2}{*}{$\begin{array}{l}\text { Variables } \\
\text { Severity of TBI }\end{array}$} & \multicolumn{2}{|c|}{ Patients with migraine } & \multicolumn{2}{|c|}{ Patients without migraine } & \multicolumn{2}{|c|}{ Compared with non-migraine } \\
\hline & Event & Rate & Event & Rate & Crude HR $^{*}(95 \% \mathrm{Cl})$ & Adjusted HR* $(95 \% \mathrm{Cl})$ \\
\hline Mild & 119 & 2.30 & 265 & 0.61 & $3.22(2.57$ to 4.03$) \dagger$ & $2.49(1.93$ to 3.20$) \dagger$ \\
\hline Severe & 13 & 0.25 & 21 & 0.05 & $5.15(2.43$ to 10.94$) \dagger$ & $3.63(1.58$ to 8.37$) \S$ \\
\hline
\end{tabular}

Rate refers to incidence rate per 1000 person-years.

*Model adjusted for age, gender, income, urbanisation level and relevant comorbidities (hypertension, diabetes mellitus, hyperlipidaemia, coronary artery disease, liver cirrhosis, mental disorders, obesity, tobacco use disorder and alcohol-attributed disease).

$\dagger P<0.001$.

$\ddagger \mathrm{P}=0.005$.

$\S \mathrm{P}=0.002$.

TBI, traumatic brain injury.

increased by limiting the analyses to patients whose claims data included at least two outpatient diagnoses. In some rare cases of TBI, the patient may only visit the emergency department without undergoing further follow-up at the outpatient department. In Taiwan, the medical treatment is easy to access at a low cost. Therefore, patients with TBI always receive initial treatment at the emergency department and undergo regular follow-up visits at the outpatient department. Second, some data needed to determine the severity of migraine or TBI were not available in this study, including the mechanism of TBI (ie, falls, motor vehicle accidents, sports injuries and violence),${ }^{46}$ the International Headache Society criteria, results of brain CT (location, size or type), associated injuries and Glasgow Coma Scale. ${ }^{47}$ Third, TBI events might have been overestimated in patients with migraine with comorbid psychiatric disorders, who consume more health resources compared with those without comorbid psychiatric disorders. Comorbid patients would be expected to seek medical services even when the severity of TBI is relatively low. ${ }^{38}$ Additionally, most inhabitants of Taiwan are ethnic Chinese; further studies are needed to investigate the association between migraine and TBI risk in other ethnicities.

In conclusion, this population-based cohort study indicated that patients with migraine have significantly higher than normal risk of developing TBI. Additionally, migraine mostly affects the development of severe TBI relative to other grades of TBI. Register-Mihalik $e t$ $a t^{48}$ have shown the baseline headache associated with increased incidence but also severity of concussion. Thus, migraine may be a poor contributor to TBI. However, further detailed studies are needed to explore the underlying mechanisms of this association. The association between change in brain ion homeostasis or excitatory amino acids and severity of advanced neuronal damage needs further elucidation. Migraine-targeted treatment modalities may help reduce morbidity and mortality associated with TBI.

Author affiliations

${ }^{1}$ Department of Neurosurgery, Qinghe County Central Hospital of Hebei Province, Qinghe, Hebei, China
${ }^{2}$ Department of Dermatology, Kaohsiung Veterans General Hospital, Kaohsiung, Taiwan

${ }^{3}$ Department of Nursing, Shu-Zen Junior College of Medicine and Management, Kaohsiung, Taiwan

${ }^{4}$ Graduate Institute of Medicine, College of Medicine, Kaohsiung Medical University, Kaohsiung, Taiwan

${ }^{5}$ Department of Surgery, Kaohsiung Medical University Hospital,Kaohsiung Medical University, Kaohsiung, Taiwan

${ }^{6}$ Department of Neurosurgery, Zaozhuang Municipal Hospital, Zaozhuang,

Shandong, China

${ }^{7}$ Department of Neurosurgery, The Affiliated Zhangjiagang Hospital of Soochow University, Zhangjiagang, Jiangsu, China

${ }^{8}$ Department of Psychiatry, Kaohsiung Chang Gung Memorial Hospital and Chang Gung University College of Medicine, Kaohsiung, Taiwan

${ }^{9}$ Department of Cardiology, Hebei Quyang Renji Hospital, Quyang, Hebei, China ${ }^{10}$ Division of Neurosurgery, Department of Surgery, Kaohsiung Medical University Hospital, Kaohsiung, Taiwan

${ }^{11}$ Department of Surgery, School of Medicine, College of Medicine, Kaohsiung Medical University, Kaohsiung, Taiwan

Contributors $\mathrm{Y}-\mathrm{YL}$ and Q-RW were equal contributors in this study. $\mathrm{HQ}$ and $\mathrm{Y}-\mathrm{JS}$ were equal contributors in this study. The authors' individual contributions are outlined as follows. Substantial contributions to conception and design: Q-RW, Y-YL, Y-JS, HQ, LZ, M-KW, C-LZ and C-HW. Drafting and revising the article critically for important intellectual content: Q-RW, Y-YL, Y-JS, HQ and C-HW. Final approval of the version to be published: $\mathrm{Q}-\mathrm{RW}, \mathrm{Y}-\mathrm{YL}$ and $\mathrm{C}-\mathrm{HW}$.

Funding This study was supported by grants from the Kaohsiung Medical University Hospital (KMUH105- 5M17 and KMUH106- 6T09).

Disclaimer The funders had no role in the study design, data collection and analysis, design to publish or preparation of the manuscript.

Competing interests None declared.

Patient consent for publication Not required.

Provenance and peer review Not commissioned; externally peer reviewed.

Data availability statement All data relevant to the study are included in the article.

Open access This is an open access article distributed in accordance with the Creative Commons Attribution Non Commercial (CC BY-NC 4.0) license, which permits others to distribute, remix, adapt, build upon this work non-commercially, and license their derivative works on different terms, provided the original work is properly cited, appropriate credit is given, any changes made indicated, and the use is non-commercial. See: http://creativecommons.org/licenses/by-nc/4.0/.

\section{REFERENCES}

1. Hyder AA, Wunderlich CA, Puvanachandra P, et al. The impact of traumatic brain injuries: a global perspective. NeuroRehabilitation 2007;22:341-53. 
2. $\mathrm{Yu} \mathrm{H}$, Watt $\mathrm{H}$, Mohan $\mathrm{S}$. The negative impact of traumatic brain injury (TBI) on bone in a mouse model. Brain Injury 2014;28:244-51.

3. Langlois JA, Rutland-Brown W, Wald MM. The epidemiology and impact of traumatic brain injury. Journal of Head Trauma Rehabilitation 2006;21:375-8.

4. Consensus conference. Consensus conference. rehabilitation of persons with traumatic brain injury. $\mathrm{NIH}$ consensus development panel on rehabilitation of persons with traumatic brain injury. JAMA 1999;282:974-83.

5. Feigin VL, Bennett DA. Handbook of clinical neuroepidemiology. New York: Nova Science Publishers, 2007.

6. Donders J, Warschausky S. Neurobehavioral outcomes after early versus late childhood traumatic brain injury. $J$ Head Trauma Rehabil 2007;22:296-302.

7. Mock C, Quansah R, Krishnan R, et al. Strengthening the prevention and care of injuries worldwide. The Lancet 2004;363:2172-9.

8. The Lancet Neurology. Traumatic brain injury: time to end the silence. The Lancet Neurology 2010;9.

9. Lucas S, Hoffman JM, Bell KR, et al. Characterization of headache after traumatic brain injury. Cephalalgia 2012;32:600-6.

10. Nampiaparampil DE. Prevalence of chronic pain after traumatic brain injury: a systematic review. JAMA 2008;300:711-9.

11. Gil-Gouveia R, Oliveira AG, Martins IP. Cognitive dysfunction during migraine attacks: a study on migraine without aura. Cephalalgia 2015;35:662-74.

12. Anderson $\mathrm{K}$, Tinawi $\mathrm{S}$, Lamoureux $\mathrm{J}$, et al. Detecting migraine in patients with mild traumatic brain injury using three different headache measures. Behav Neurol 2015;2015:693925

13. Monteith TS, Borsook D. Insights and advances in post-traumatic headache: research considerations. Curr Neurol Neurosci Rep 2014;14:428.

14. Gordon KE, Dooley JM, Wood EP. Is migraine a risk factor for the development of concussion? Br J Sports Med 2006;40:184-5.

15. CH W, Tsai TH, YF S, et al. Traumatic brain injury and substance related disorder: a 10-year nationwide cohort study in Taiwan. Neural Plast 2016

16. Wu C-H, Zhang Z-H, Wu M-K, et al. Increased migraine risk in osteoporosis patients: a nationwide population-based study. Springerplus 2016:5:1378

17. Yang C-P, Chen Y-T, Fuh J-L, et al. Migraine and risk of ocular motor cranial nerve palsies: a nationwide cohort study. Ophthalmology 2016:123:191-7.

18. Tung Y-C, Tu H-P, Tsai W-C, et al. Increased incidence of herpes zoster and postherpetic neuralgia in adult patients following traumatic brain injury: a nationwide population-based study in Taiwan. PLoS One 2015;10:e0129043.

19. Coronado VG, Xu L, Basavaraju SV, et al. Surveillance for traumatic brain injury-related deaths--United States, 1997-2007. MMWR Surveill Summ 2011:60:1-32.

20. Wu C-L, Kor C-T, Chiu P-F, et al. Long-Term renal outcomes in patients with traumatic brain injury: a nationwide population-based cohort study. PLoS One 2017;12:e0171999.

21. Shi H-Y, Hwang S-L, Lee K-T, et al. Temporal trends and volumeoutcome associations after traumatic brain injury: a 12-year study in Taiwan. J Neurosurg 2013;118:732-8.

22. Chi Y-C, Wu H-L, Chu C-P, et al. Traumatic brain injury and affective disorder: a nationwide cohort study in Taiwan, 2000-2010. J Affect Disord 2016;191:56-61.

23. Chung K-H, Li C-Y, Kuo S-Y, et al. Risk of psychiatric disorders in patients with chronic insomnia and sedative-hypnotic prescription: a nationwide population-based follow-up study. J Clin Sleep Med 2015:11:543-51.

24. Lee $\mathrm{Y}-\mathrm{K}$, Lee $\mathrm{C}-\mathrm{W}$, Huang $\mathrm{M}-\mathrm{Y}$, et al. Increased risk of ischemic stroke in patients with mild traumatic brain injury: a nationwide cohort study. Scand J Trauma Resusc Emerg Med 2014;22:66.

25. HY LCY, YL C, et al. Incorporating development stratification of Taiwan townships into sampling design of large scale health interview survey. J Health Manag 2006;4:1-22.
26. Yang Y-J, Chien W-C, Chung C-H, et al. Risk of erectile dysfunction after traumatic brain injury: a nationwide population-based cohort study in Taiwan. Am J Mens Health 2018;12:913-25.

27. Wang S-H, Wang J, Lin Y-S, et al. Increased risk for incident thyroid diseases in people with psoriatic disease: a cohort study. J Am Acad Dermatol 2019;80:1006-12.

28. HS Y, HP T, Hong $\mathrm{CH}$, et al. Lifetime increased risk of adult onset atopic dermatitis in adolescent and adult patients with food allergy. Int J Mol Sci 2016;18.

29. Lin $\mathrm{C}-\mathrm{H}$, Chang W-C, Kuo C-N, et al. A population-based five-year study on the risk of stroke in patients with osteoporosis in Taiwan. Bone 2015;72:9-13.

30. Scopaz KA, Hatzenbuehler JR. Risk modifiers for concussion and prolonged recovery. Sports Health 2013;5:537-41.

31. Lau B, Lovell MR, Collins MW, et al. Neurocognitive and symptom predictors of recovery in high school athletes. Clinical Journal of Sport Medicine 2009;19:216-21.

32. Lauritzen M, Dreier JP, Fabricius M, et al. Clinical relevance of cortical spreading depression in neurological disorders: migraine, malignant stroke, subarachnoid and intracranial hemorrhage, and traumatic brain injury. J Cereb Blood Flow Metab 2011;31:17-35.

33. Eckner JT, Seifert T, Pescovitz A, et al. Is migraine headache associated with concussion in athletes? A Case-Control study. Clinical Journal of Sport Medicine 2017;27:266-70.

34. Gil-Gouveia R, Oliveira AG, Martins IP. Assessment of cognitive dysfunction during migraine attacks: a systematic review. J Neurol 2015;262:654-65.

35. Chuang C-S, Lin C-L, Lin M-C, et al. Migraine and risk of dementia: a nationwide retrospective cohort study. Neuroepidemiology 2013;41:139-45

36. Royall DR, Lauterbach EC, Cummings JL, et al. Executive control function: a review of its promise and challenges for clinical research. A report from the Committee on research of the American neuropsychiatric association. J Neuropsychiatry Clin Neurosci 2002;14:377-405.

37. Nordström A, Edin BB, Lindström S, et al. Cognitive function and other risk factors for mild traumatic brain injury in young men: nationwide cohort study. BMJ 2013;346.

38. Antonaci F, Nappi G, Galli F, et al. Migraine and psychiatric comorbidity: a review of clinical findings. $J$ Headache Pain 2011;12:115-25.

39. Silberstein SD, Lipton RB, Breslau N. Migraine: association with personality characteristics and psychopathology. Cephalalgia 1995;15:358-69.

40. Mongini F, Keller R, Deregibus A, et al. Personality traits, depression and migraine in women: a longitudinal study. Cephalalgia 2003;23:186-92.

41. Liao C-C, Chiu W-T, Yeh C-C, et al. Risk and outcomes for traumatic brain injury in patients with mental disorders. $J$ Neurol Neurosurg Psychiatry 2012;83:1186-92.

42. Fann JR, Leonetti A, Jaffe K, et al. Psychiatric illness and subsequent traumatic brain injury: a case control study. J Neurol Neurosurg Psychiatry 2002;72:615-20.

43. Kuny S, Stassen $\mathrm{HH}$. Cognitive performance in patients recovering from depression. Psychopathology 1995;28:190-207.

44. Golinkoff M, Sweeney JA. Cognitive impairments in depression. Affect Disord 1989;17:105-12.

45. M XL F, MM W, et al. Traumatic brain injury in the United States: emergency department visits, hospitalizations and deaths 2002-2006. Atlanta, GA: Centers for Disease Control and Prevention, National Center for Injury Prevention and Control, 2010.

46. Yang $\mathrm{W}-\mathrm{H}$, Chen $\mathrm{P}-\mathrm{C}$, Wang $\mathrm{T}-\mathrm{C}$, et al. Endocrine dysfunction following traumatic brain injury: a 5-year follow-up nationwide-based study. Sci Rep 2016;6:32987.

47. Chou Y-C, Yeh C-C, Hu C-J, et al. Risk and mortality of traumatic brain injury in stroke patients: two nationwide cohort studies. J Head Trauma Rehabil 2014:29:514-21.

48. Register-Mihalik J, Guskiewicz KM, Mann JD, et al. The effects of headache on clinical measures of neurocognitive function. Clinical Journal of Sport Medicine 2007;17:282-8. 